\title{
Severe Encephalopathy, Lactic Acidosis, Vegetative Instability and Neuropathy with 5-Fluorouracil Treatment - Pyrimidine Degradation Defect or Beriberi?
}

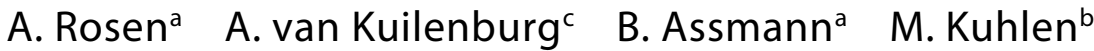 \\ A. Borkhardt ${ }^{\text {b }}$ \\ Departments of a General Pediatrics, and ${ }^{\mathrm{b} P e d i a t r i c}$ Oncology, Hematology and \\ Immunology, University Children Hospital Düsseldorf, Düsseldorf, Germany; \\ 'Laboratory Genetic Metabolic Diseases, Academic Medical Center of the \\ University of Amsterdam, Amsterdam, The Netherlands
}

\section{Key Words}

Beriberi · 5-FU · Pyrimidine degradation defect

\begin{abstract}
We present the case of a 19-year-old female with nasopharyngeal carcinoma, who received two courses of chemotherapy with 5 -fluorouracil $(5-\mathrm{FU})$ in combination with folic acid and cisplatin. Upon developing esophageal strictures in the course of her radiotherapy, she required total parenteral nutrition. In the course of therapy, the patient developed severe multisystem failure with encephalopathy, lactic acidosis, vegetative instability and neuropathy. The treatment with 5-FU can lead to severe toxicity due to enzyme deficiencies in the degradation of pyrimidines, but it can also lead to thiamine deficiency with the classic symptoms of beriberi. Beriberi is a rare disorder, usually attributed to malnutrition or alcoholism. 5-FU has been shown to induce thiamine depletion. Reduced food intake or total parenteral nutrition devoid of vitamin supplements may aggravate symptoms. We were unable to find a genetic cause for increased 5-FU toxicity in our patient, ruling out deficiencies of dihydropyrimidine dehydrogenase, dihydropyrimidinase or $\beta$-ureidopropionase and double-strand break repair deficits. We come to the conclusion that, even without any definable enzyme deficiency, treatment with 5-FU can lead to high toxicity due to thiamine deficiency if vitamin supplementation is not undertaken.
\end{abstract}




\section{Introduction}

Vitamin $\mathrm{B}_{1}$, or thiamine, is an important dietary factor for the human metabolism. The phosphorylated form, thiamine pyrophosphate (TPP), serves as cofactor for several vital metabolic processes, most importantly the conversion of pyruvate to acetyl coenzyme A and the conversion of $\alpha$-ketoglutarate to succinyl coenzyme $\mathrm{A}$ in the citric acid cycle and several chemical reactions in the pentose-phosphate pathway. The human body requires between 0.3 and $1.7 \mathrm{mg}$ of vitamin $\mathrm{B}_{1}$ per day, depending on age and gender. Vitamin $\mathrm{B}_{1}$ is present in yeast, soy products, legumes, cereals and meat.

Without regular intake of this vitamin, energy metabolism in organs such as the brain or the heart is compromised, leading to symptoms of beriberi. 'Beriberi' is a Singhalese term, roughly translating to 'I cannot, I cannot', which aptly describes the loss of body functions in the course of the disease. The symptoms of beriberi are divided into two forms, which can be seen in combination or isolated from each other:

(1) 'Dry beriberi', consisting of peripheral neuropathy and Wernicke's encephalopathy, a combination of ophthalmoparesis, diplopia, horizontal nystagmus, reflex disorders, confusion, disorientation, apathy, somnolence, ataxia, dysdiadochokinesia, dysarthria, dysphagia, insomnia, psychosis and vegetative symptoms such as hypotension, hypothermia or hyperhydrosis.

(2) 'Wet beriberi', consisting of cardiomyopathy, heart failure, cardiomegaly, peripheral edemas and tachycardia.

Vitamin $B_{1}$ deficiency is a rare disorder, usually observed in patients with alcoholism or with severe malnutrition. There are, however, cases of iatrogenic vitamin $\mathrm{B}_{1}$ deficiency, where long-term treatment with total parenteral nutrition (TPN) devoid of thiamine induced symptoms of beriberi in pediatric populations [1-3]. Four general causes can be identified, which can lead to symptoms of thiamine deficiency either by themselves or in combination:

(1) Reduced thiamine intake (due to fasting, alcoholism, general malnutrition, inactivation of thiamine in food by cooking or through inactivating factors contained in certain fish, tannins or coffee, or iatrogenic as described above).

(2) Reduced thiamine absorption (short bowel syndrome, celiac disease, chronic inflammatory bowel disease).

(3) Increased thiamine excretion (diarrhea, dialysis, diuretic treatment).

(4) Increased thiamine metabolism (pregnancy, fever, activity, iatrogenic).

Patients receiving 5-fluorouracil (5-FU) have shown to have an increased thiamine metabolism and subsequently low thiamine levels $[4,5]$. Several reports describe cases of increased 5-FU toxicity due to enzyme mutations in the pyrimidine degradation pathway. The most frequent cause is a defect of dihydropyrimidine dehydrogenase (DPD), less common causes include defects of the enzyme dihydropyrimidinase and $\beta$ ureidopropionase [6-8]. 


\section{Case Presentation}

A 19-year-old female Caucasian patient was diagnosed with Hodgkin's lymphoma in December 2004. She was treated with 2 blocks of OPPA (vincristine, procarbazine, prednisone and doxorubicin), 2 blocks of COPP (cyclophosphamide, vincristine, procarbazine and prednisone) and consolidating radiotherapy with a total dose of 19.8 Gy until May 2005. After a good recovery and uneventful checkups from 2005 until 2009, she was diagnosed with a nasopharyngeal carcinoma including cervical lymph node metastases in May 2009.

Starting in June 2009, we began a chemotherapy treatment according to the NPC-2003-GPOH multicentric study for the treatment of nasopharyngeal carcinoma in children and adolescents (nasopharyngeal carcinoma trial 2003 of the German Society for Pediatric Oncology and Hematology). The initial treatment in this protocol consists of 3 blocks of cisplatin $100 \mathrm{mg} / \mathrm{m}^{2}$ on day 1 and 5 -FU $1,000 \mathrm{mg} / \mathrm{m}^{2} /$ day for 5 consecutive days as well as 6 doses of folic acid $25 \mathrm{mg} / \mathrm{m}^{2}$ every $6 \mathrm{~h}$ [9]. After the first 5-day course of this combination, the patient developed massive mucositis, peripheral edemas, paresthesia and electrolyte imbalances and had to be hospitalized for 3 weeks until recovery.

The second course of chemotherapy was started in July 2009. In the beginning of August, 2 weeks after the end of the second block of chemotherapy, our patient again developed severe symptoms of even more pronounced toxicity: esophageal strictures, massive mucositis, peripheral edemas, myelosuppression, electrolyte imbalances, lactic acidosis, renal insufficiency, hepatopathy with hyperbilirubinemia and coagulation deficits, hypoalbuminemia with ascites, pericardial and pleural effusion as well as a complex neuropathy with hallucinations, reduced vision, miosis, ptosis, slow pupil reactions, a reduced state of consciousness, slurred speech, a vegetative crisis with problems to control blood pressure and body temperature, polyradiculitis, peripheral loss of sensibility in the lower legs and paresthesia of both hands and feet.

We suspected encephalitis or an antineuronal autoimmune process and began treatment with intravenous acyclovir, immunoglobulins and a substitution of sodium bicarbonate and electrolytes. Additionally, the patient also received thiamine infusions for treating a suspected thiamine deficiency. The blood results later revealed a concentration of $18.1 \mu \mathrm{g} / \mathrm{l} \mathrm{TPP}$, normal values ranging from 28.0 to 85.0. The patient showed signs of improvement within hours after this multimodal approach and recovered within several days.

Due to these complications, we performed extensive work-up: cranial MRIs as well as an EEG showed no pathological findings. Laboratory tests of the cerebrospinal fluid showed elevated lactate and protein, but no growth of bacteria, normal numbers of red and white blood cells and no viruses in the standard polymerase chain reaction tests. We were also able to rule out paraneoplastic reactions with antineuronal antibodies (Yo, $\mathrm{Hu}, \mathrm{Ri}, \mathrm{Ma}$ 1, Ma2, CV2, amphysin). Due to the close proximity of the symptom onset after 5-FU treatment, we suspected a defect in the metabolism of pyrimidines. A normal activity of DPD was detected in the peripheral blood mononuclear cells, thus ruling out DPD deficiency $(8.0 \mathrm{nmol} / \mathrm{mg} / \mathrm{h}$ compared to $9.9 \pm 2.8 \mathrm{nmol} / \mathrm{mg} / \mathrm{h}$ observed in controls $)$. A complete deficiency of dihydropyrimidinase or $\beta$-ureidopropionase was ruled out by normal results in the analysis of pyrimidine degradation metabolites in urine samples (dihydrouracil $<4 \mu \mathrm{mol} / \mathrm{l}$, dihydrothymine $<1 \mu \mathrm{mol} / \mathrm{l}, \mathrm{N}-\mathrm{C}$ - $\beta$-alanine $7 \mu \mathrm{mol} / \mathrm{l}, \mathrm{N}-\mathrm{C}$ - $\beta$-aminoisobutyric acid $1 \mu \mathrm{mol} / \mathrm{l}$, uracil $6 \mu \mathrm{mol} / \mathrm{l}$, thymine $<0.4$ $\mu \mathrm{mol} / 1$, 5 -hydroxymethyluracil $<0.5 \mu \mathrm{mol} / \mathrm{l}$ ). Finally, skin fibroblast cultures revealed no double-strand break repair deficits or abnormalities in the flux analysis of radioactively labelled deoxyuridine and 5fluoro-2'-deoxyuridine. We were thus unable to find a genetic cause for increased 5-FU toxicity in our patient.

After this episode, we decided not to administer 5-FU again and opted for a combination of epirubicin, paclitaxel and cisplatin for the third course of chemotherapy in August 2009. This course was tolerated much better than the previous two and in September of 2009, we were able to begin adjuvant radiation therapy, which was also well tolerated. The patient's esophageal strictures were, however, further exacerbated by radiation therapy. We performed endoscopic dilatation of the esophagus and provided the patient with TPN during the weeks of radiation therapy, as she was not able to take in food orally. For the first 6 weeks of intravenous alimentation, the patient also received vitamin supplementation with both fat- and water-soluble vitamins, including vitamin $B_{1}$. After a second dilatation of the esophagus in October 2009, the patient again required TPN for a period of 11 days. This time, however, vitamin supplements were not added. After the end of the 6-week course of radiation, the patient was able to take in food orally again and was discharged from the hospital. 
One week after the end of radiotherapy, she was admitted to the oncology ward again due to radiation-induced laryngitis. She had not been able to take in solid foods for some days and had lost 1.6 $\mathrm{kg}$ over the course of 5 days. While on our ward, she again developed neurologic symptoms, especially reduced vigilance and drowsiness and began to vomit uncontrollably. Laboratory tests showed an acidosis with lactate of $6.31 \mathrm{mmol} / \mathrm{l}$ (normal value $<2.20$ ). This time, we immediately suspected a thiamine deficiency and administered intravenous thiamine at a dosage of $100 \mathrm{mg}$ over the course of $1 \mathrm{~h}$. Laboratory tests later confirmed this suspicion, showing a TPP in the EDTA blood drawn before thiamine substitution of $8.1 \mu \mathrm{g} / \mathrm{l}$ (normal values $28.0-85.0$ ).

The patient initially improved neurologically, but became unconscious again after about half an hour, with a blood sugar of $28 \mathrm{mg} / \mathrm{dl}$ (normal values 60-100). After an infusion of glucose solution $(50 \%)$ and stopping the thiamine infusion, the patient improved rapidly with normalization of laboratory findings and neurological status. Over the course of the next 5 days, we continued low-dose thiamine substitution under close surveillance of the blood sugar and saw a continuous improvement of the patient's condition. She began taking solid foods again and was prescribed oral multivitamin supplementation. Until today, similar episodes of symptomatic thiamine deficiency have not occurred again. The patient is currently living in remission and is doing remarkably well.

\section{Discussion}

In retrospect, it can be confirmed that our patient developed the classic symptoms of thiamine deficiency (beriberi) as a result of 5-FU toxicity and hypoalimentation with vitamin $\mathrm{B}_{1}$. 5-FU has been shown to aggravate or even induce thiamine deficits by increasing the cellular thiamine metabolism [10]. Studies were able to demonstrate thiamine deficiency as well as decreased activity of the thiamine-dependent enzyme transketolase after treatment with 5-FU $[4,5,11]$. Before the first episode of beriberi in August 2009, no thiamine levels were determined, but blood tests undertaken after the onset of symptoms showed low TPP, suggesting thiamine depletion during the course of chemotherapy. Despite successful treatment of the complications by a multimodal approach which included thiamine infusion, we were at that point not able to exactly determine the cause of the clinical symptoms.

A genetic and/or biochemical explanation for the increased toxicity of 5-FU in our patient was not found. Patients with a deficit in the metabolism of pyrimidines frequently experience increased toxicity after 5 -FU treatment $[8,12]$. In our patient, extensive biochemical and genetic work-up did not reveal any known defect in the pyrimidine pathway indicating the existence of other, yet unknown reasons for this severe spectrum of side effects.

After developing esophageal strictures, which limited the oral intake of food and led to a hypoalimentation with vitamin $B_{1}$, the patient developed a second episode of thiamine deficiency. This time, the symptoms were immediately attributed to a possible thiamine deficiency and correctly treated with intravenous infusion of thiamine. However, due to the depleted thiamine levels, the substitution of thiamine induced a state of hypoglycemia. 'The phenomenon of post-i.v. thiamine hypoglycemia is observed not infrequently in (...) thiamine deficiency' [13].

There are several lessons to be learned from this case:

(1) The combination of lactic acidosis, heart failure, central and peripheral neuropathy are highly suggestive of a thiamine deficiency in the setting of an unbalanced diet and/or aggressive medication. 
(2) When administering 5-FU to patients, thiamine levels should be monitored and thiamine supplemented upon signs of deficiency.

(3) Long-term TPN should always contain vitamin supplementation.

(4) Thiamine supplementation in patients with extensive deficiencies can induce hypoglycemia and should therefore be accompanied by glucose-infusions.

(5) Even without genetic defects of pyrimidine degradation, 5-FU treatment can cause exceptional toxicity which may be the result of thiamine deficiency.

In conclusion, the treatment of cancer patients with 5-FU can lead to thiamine deficiency with the classic symptoms of beriberi and may be aggravated by reduced oral food intake or TPN devoid of vitamin supplementation. According to a literature review, thiamine deficiency in patients receiving TPN or having been treated with 5-FU is not uncommon, yet probably underdiagnosed $[14,15]$. Knowledge of this important side effect of 5-FU treatment may help to prevent severe complications in future patients. Testing for genetic defects in the metabolism of pyrimidines should be undertaken before starting the treatment. However, clinicians should be alert for the development of severe thiamine deficiency even if no genetic defect in the pyrimidine degradation pathway can be documented.

\section{Consent}

Written informed consent was obtained from the patient for publication of this case report. A copy of the written consent is available for review from the Editor-in-Chief.

\section{Acknowledgements}

The authors acknowledge the important contributions of the following people to this case report: The patient and her family for offering their consent to have this case report published. Dr. R. Wessalowski and Dr. G. Janßen for their encouragement and support. The nurses of the childhood oncology ward KK04 and the outpatient department KA04. Dr. C. Rübe and her team for their contributions to the laboratory workup. Dr. Ö. Degistirici and his team for their help with the fibroblast cultures. Dr. T. el Scheich and Dr. J. Marquard for their editorial help.

\section{Disclosure Statement}

The authors declare that they have no competing interests. 


\section{References}

$\checkmark 1$ Blennow G: Wernicke encephalopathy following prolonged artificial nutrition. Am J Dis Child 1975;129:14561460.

2 Fattal-Valevski A, Kesler A, Sela BA, et al: Outbreak of life-threatening thiamine deficiency in infants in Israel caused by a defective soy-based formula. Pediatrics 2005;115:e233-e238.

-3 Hahn JS: Wernicke encephalopathy and beriberi during total parenteral nutrition attributable to multivitamin infusion shortage. Pediatrics 1998;101:e10.

-4 Aksoy M, Basu TK, Brient J, Dickerson JW: Thiamin status of patients treated with drug combinations containing 5-fluorouracil. Eur J Cancer 1980;16:1041-1045.

5 Basu TK, Aksoy M, Dickerson JWT: Effects of 5-flourouracil on the thiamin status of adult female rats. Chemotherapy 1979;25:70-76.

-6 Mattison LK, Soong R, Diasio RB: Implications of dihydropyrimidine dehydrogenase on 5-fluorouracil pharmacogenetics and pharmacogenomics. Pharmacogenomics 2002;3:485-492.

7 van Kuilenburg ABP, et al: Dihydropyrimidinase deficiency and severe 5-fluorouracil toxicity. Clin Cancer Res 2003;9:4363-4367.

8 van Kuilenburg ABP: Dihydropyrimidine dehydrogenase and the efficacy and toxicity of 5-fluorouracil. Eur J Cancer 2004;40:939-950.

9 Mertens R, et al: NPC-2003-GPOH Study Protocol. University Childrens Clinic of RWTH Aachen, 2003.

10 Heier MS, Dornish JM: Effect of the fluoropyrimidines 5-fluorouracil and doxifluridine on cellular uptake of thiamin. Anticancer Res 1989;9:1073-1077.

11 Kondo K, Fujiwara M, Murase M, Kodera Y, Akiyama S, Ito K, Takagi H: Severe acute metabolic acidosis and Wernicke's encephalopathy following chemotherapy with 5-fluorouracil and cisplatin: case report and review of the literature. Jpn J Clin Oncol 1996;26:234-236.

-12 Koenig H: Biochemical basis for fluorouracil neurotoxicity. Arch Neurol 1970;23:155-160.

13 Naviaux RK: Re: [metab-1] Thiamine and fall in blood glucose. E-mail to metab-l discussion group on inborn errors of metabolism, November 30th, 2010.

14 Francini-Pesenti F: Wernicke's syndrome during parenteral feeding: not an unusual complication. Nutrition 2009;25:142-146.

15 Kitamura K, Yamaguchi T, Tanaka H, et al: TPN-induced fulminant beriberi: a report on our experience and a review of the literature. Surg Today 1996;26:769-776. 